\title{
Fluxon dynamics in three stacked Josephson junctions
}

Gorria, Carlos; Christiansen, Peter Leth; Gaididei, Yuri Borisovich; Muto, Virginia; Pedersen, Niels Falsig; Sørensen, Mads Peter

Published in:

Physical Review B Condensed Matter

Link to article, DOI:

10.1103/PhysRevB.66.172503

Publication date:

2002

Document Version

Publisher's PDF, also known as Version of record

Link back to DTU Orbit

Citation (APA):

Gorria, C., Christiansen, P. L., Gaididei, Y. B., Muto, V., Pedersen, N. F., \& Sørensen, M. P. (2002). Fluxon dynamics in three stacked Josephson junctions. Physical Review B Condensed Matter, 66(17), 172503. https://doi.org/10.1103/PhysRevB.66.172503

\section{General rights}

Copyright and moral rights for the publications made accessible in the public portal are retained by the authors and/or other copyright owners and it is a condition of accessing publications that users recognise and abide by the legal requirements associated with these rights.

- Users may download and print one copy of any publication from the public portal for the purpose of private study or research.

- You may not further distribute the material or use it for any profit-making activity or commercial gain

- You may freely distribute the URL identifying the publication in the public portal 


\title{
Fluxon dynamics in three stacked Josephson junctions
}

\author{
C. Gorria, ${ }^{1,2}$ P. L. Christiansen, ${ }^{1}$ Yu. B. Gaididei, ${ }^{1,3}$ V. Muto, ${ }^{2}$ N. F. Pedersen, ${ }^{4}$ and M. P. Soerensen ${ }^{1}$ \\ ${ }^{1}$ Section of Mathematical Physics, IMM, Technical University of Denmark, DK-2800, Kgs. Lyngby, Denmark \\ ${ }^{2}$ Department of Applied Mathematics and Statistics, University of the Basque Country, E - 48080 Bilbao, Spain \\ ${ }^{3}$ Bogolyubov Institute for Theoretical Physics, 252143 Kiev, Ukraine \\ ${ }^{4}$ Department of Electric Power Engineering, Technical University of Denmark, DK-2800, Kgs. Lyngby, Denmark
}

(Received 15 July 2002; revised manuscript received 27 August 2002; published 11 November 2002)

The motion of fluxons of the same polarity in three vertically stacked Josephson junctions is studied. In this configuration the difference between exterior and interior junctions plays a more important role than in other configurations with several interior junctions. Below the Swihart velocity $c_{-}$, the coupling between junctions leads to a repulsion of the fluxons with the same polarity. Above this critical velocity a fluxon will induce radiation in the neighboring junctions, leading to a bunching of the fluxons in the stacked junctions. Using the Sakai-Bodin-Pedersen model, three coupled perturbed sine-Gordon equations are numerically studied for different values of coupling, damping, and bias parameters. In a narrow range of velocities bunching occurs. Outside this interval the fluxons split and new fluxons may be created. $I-V$ characteristics are presented.

DOI: 10.1103/PhysRevB.66.172503

PACS number(s): 74.50.+r, 05.45.Yv, 85.25.Cp

In the last decades the propagation of electromagnetic waves in long Josephson junctions has been extensively studied in order to develop useful devices for storage and transmission of information. When two long layers of superconducting material are separated by an insulating layer (junction) and overlap between Cooper pairs occurs, then tunneling of electrons through the insulating barrier takes place. The phase difference $\varphi$ between the wave functions in the two superconducting layers is governed by the perturbed sine-Gordon equation. After normalizing the spatial variable to the Josephson penetration length $\lambda_{J}$ and the time to the inverse of the plasma frequency, $\omega_{0}$, the general form of the equation is

$$
\varphi_{x x}-\varphi_{t t}-\sin \varphi=\alpha \varphi_{t}-\beta \varphi_{x x t}-\gamma
$$

Here $\alpha \varphi_{t}$ represents dissipation due to the tunneling of quasiparticles through the barrier, $\beta \varphi_{x x t}$ is the dissipation due to the surface loss in the superconductors, and $\gamma$ represents the bias current density or energy input. In this paper we do not take the influence of the surface loss into account (i.e., $\beta$ $=0$ ).

In the unperturbed case $(\alpha=\gamma=0)$ Eq. (1) allows simple single soliton solutions given by the expression

$$
\varphi(x, t)=4 \arctan \left[\exp \sigma\left(\frac{x-v t-x_{0}}{\sqrt{1-v^{2}}}\right)\right],
$$

called a fluxon $(\sigma=1)$ or an antifluxon $(\sigma=-1)$, depending on the polarity. Several perturbation methods have been developed to study this model in detail. ${ }^{1}$

Stacking the junctions may increase the usability of these devices. A realistic theory describing a general system of $N$ junctions was deduced by Sakai, Bodin, and Pedersen ${ }^{2}$ from the Maxwell, London and Josephson equations. The basic ideas for the two junctions case were established before by Mineev et al. ${ }^{3}$ The electromagnetic interaction between adjacent junctions is represented by a coupling constant $S$. In this paper the particular case of three junctions is investi- gated (see Fig. 1). It is the simplest generalizable case because it takes into account the difference between the behavior of the exterior and interior junctions. The first and third junctions are coupled only to one neighboring junction while the second junction is coupled to its two neighbor junctions below and above. The governing equations for the three stacked junctions after normalization of the coupling constant $S$, where $-0.5<S \leqslant 0$, are

$$
\begin{gathered}
J_{1}=\frac{1}{1-2 S^{2}}\left[\varphi_{1, x x}-S \varphi_{2, x x}+S^{2}\left(\varphi_{3, x x}-\varphi_{1, x x}\right)\right], \\
J_{2}=\frac{1}{1-2 S^{2}}\left[\varphi_{2, x x}-S\left(\varphi_{1, x x}+\varphi_{3, x x}\right)\right], \\
J_{3}=\frac{1}{1-2 S^{2}}\left[\varphi_{3, x x}-S \varphi_{2, x x}+S^{2}\left(\varphi_{1, x x}-\varphi_{3, x x}\right)\right],
\end{gathered}
$$

where $J_{i}=\varphi_{i, t t}+\alpha_{i} \varphi_{i, t}+\sin \varphi_{i}+\gamma_{i}$. To produce junctions with identical properties is technically difficult in practice;

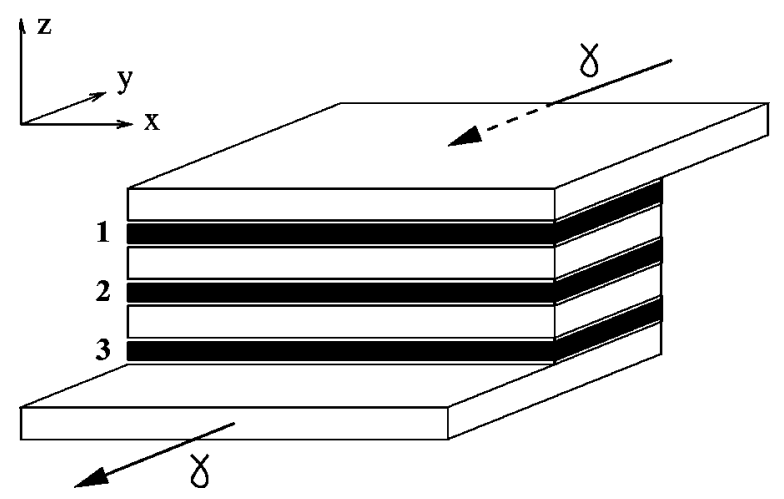

FIG. 1. Structure of the stack of four superconductors and three intermediate junctions $(1,2,3)$. Uniform external bias is applied along the system. 
however, we assume identical parameters in all equations in order to simplify the model $\left(\alpha_{i}=\alpha, \gamma_{i}=\gamma, i=1,2,3\right)$.

We shall investigate the propagation of one fluxon in each junction $[\sigma=1$ in Eq. (2)] excited by an applied external bias current $\gamma$. This external force drives the three fluxons in the same direction. ${ }^{1}$ Due to the symmetry of the system, ${ }^{4,5}$ $\varphi_{1}=\varphi_{3}$, Eqs. (3) reduce to

$$
\begin{aligned}
& J_{1}=\frac{1}{1-2 S^{2}}\left(\varphi_{1, x x}-S \varphi_{2, x x}\right), \\
& J_{2}=\frac{1}{1-2 S^{2}}\left(\varphi_{2, x x}-2 S \varphi_{1, x x}\right) .
\end{aligned}
$$

The coupling constant $2 S$ of the second junction forces its corresponding fluxon to travel slightly separated from its neighbors in the first and third junctions. ${ }^{6}$ This condition markedly perturbs the shape of the fluxons and leads to different propagation states, depending on the magnitude of the external bias current. The behavior differs clearly from the case of two identical junctions where the two equations become symmetric. $5,7,8$

In order to determine the motion of the centers of mass of the fluxons, we introduce the corresponding kinetic energy $T$ and the potential energy $W=U+U_{i n t}$ of the coupled perturbed system, Eqs. (3):

$$
\begin{gathered}
T=\int\left(\varphi_{1, t}^{2}+\frac{1}{2} \varphi_{2, t}^{2}\right) d x \\
U=\int\left(\frac{\varphi_{1, x}^{2}+\frac{1}{2} \varphi_{2, x}^{2}}{1-2 S^{2}}+3-2 \cos \varphi_{1}-\cos \varphi_{2}\right. \\
\left.-\gamma\left(2 x \varphi_{1, x}+x \varphi_{2, x}\right)\right) d x \\
U_{i n t}=\frac{-2}{1-2 S^{2}} \int \varphi_{1, x} \varphi_{2, x} d x .
\end{gathered}
$$

For unperturbed conditions $(\alpha=\gamma=0)$ the total energy of the system, $E=T+W$, will be preserved $(d E / d t=0)$. When driving and damping come into the equations the stable states will coincide with the local minima of the potential energy $W$. Here $U_{\text {int }}$, representing the interaction between fluxons, is the only term in $W$ which depends on the distance between fluxons. Using the adiabatic approximation, ${ }^{1}$ we observe that the effect of the coupling is to repel equal-polarity fluxons and to attract opposite-polarity ones, as shown in Refs. 9 and 10. However, interesting bunching phenomena of equal-polarity fluxons ${ }^{7,9,11}$ may occur for certain ranges of high speeds above the Swihart velocity. ${ }^{12}$

The characteristic velocities, corresponding to the three linear modes of the plasma waves, ${ }^{13,14}$ are obtained by substituting a periodic-wave-type function $\varphi_{j}=A_{j} e^{i(k x-\omega t)}$ into the linear equation obtained by setting $\alpha=\gamma=0$ and linear-
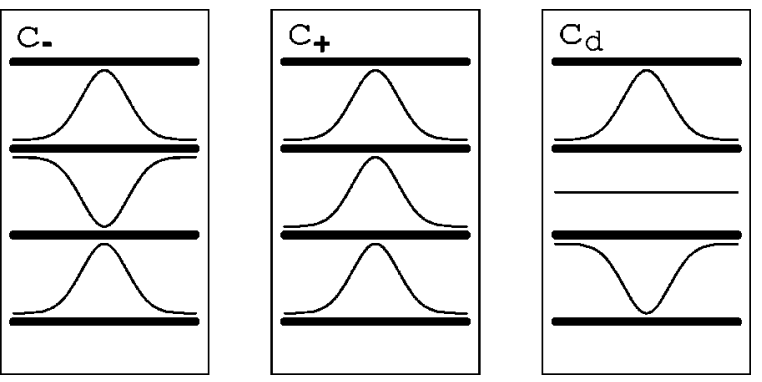

FIG. 2. Schematic drawing of the antiphase $\left(c_{-}\right)$, in-phase $\left(c_{+}\right)$, and decoupled $\left(c_{d}\right)$ modes.

izing $\sin \varphi_{i} \approx \varphi_{i}, i=1,2,3$ in Eqs. (3). The existence of these characteristic velocities for two junctions case was investigated numerically in Ref. 15. For three junctions the expressions for these three velocities are $c_{\mp}=1 / \sqrt{1 \mp \sqrt{2} S}$ and $c_{d}$ $=1$. The values of $c_{-}, c_{+}$, and $c_{d}$ coincide with the maximum velocities of the fluxon-antifluxon-fluxon (f-a-f), fluxon-fluxon-fluxon (f-f-f) and fluxon-0-antifluxon (f-0-a) configurations, respectively. Similarly to Ref. 16 , these configurations are shown schematically in Fig. 2.

The aim of the present study is to understand the dynamics of the fluxons on three damped and biased junctions, one fluxon in each junction, driven in the high-velocity regime. Quite recently the interaction of fluxons in the case of two and three junctions has been investigated by Goldobin et al. ${ }^{4,5}$ where it was numerically shown that several fluxons in one of the junctions may bunch due to Cherenkov emission in the adjacent junctions above the Swihart velocity $c_{-}$.
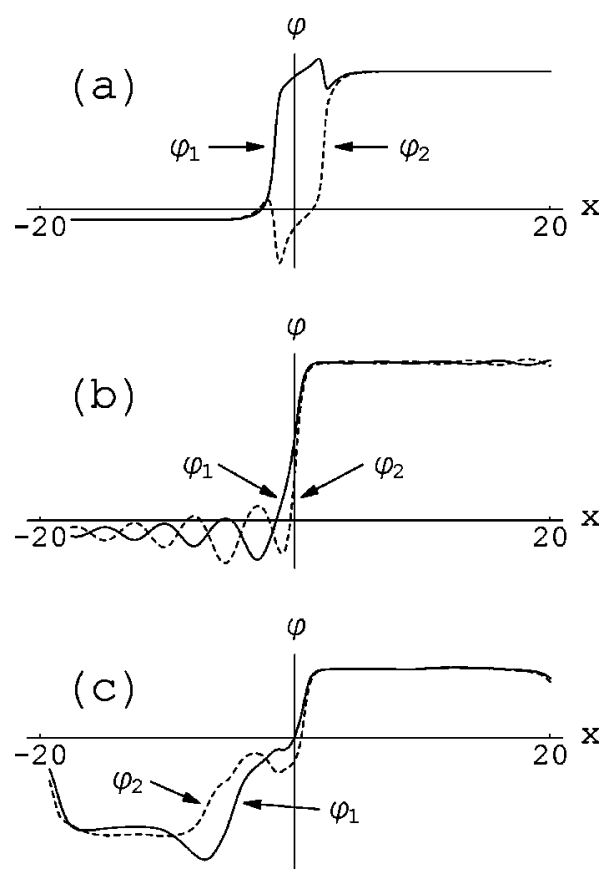

FIG. 3. Behavior of fluxons $\varphi_{1}$ (solid curve) and $\varphi_{2}$ (dashed curve) for coupling $S=-0.2$, damping $\alpha=0.1$, and bias current: (a) $\gamma=0.43$ fluxons split with velocities $v_{1}=0.868$ for $\varphi_{1}$ and $v_{2}$ $=0.88$ for $\varphi_{2}$, (b) $\gamma=0.44$ bunched fluxons with velocity $v_{1}=v_{2}$ $=1.118$, and (c) $\gamma=0.69$ creation of new fluxon-antifluxon pair due to excess energy. 

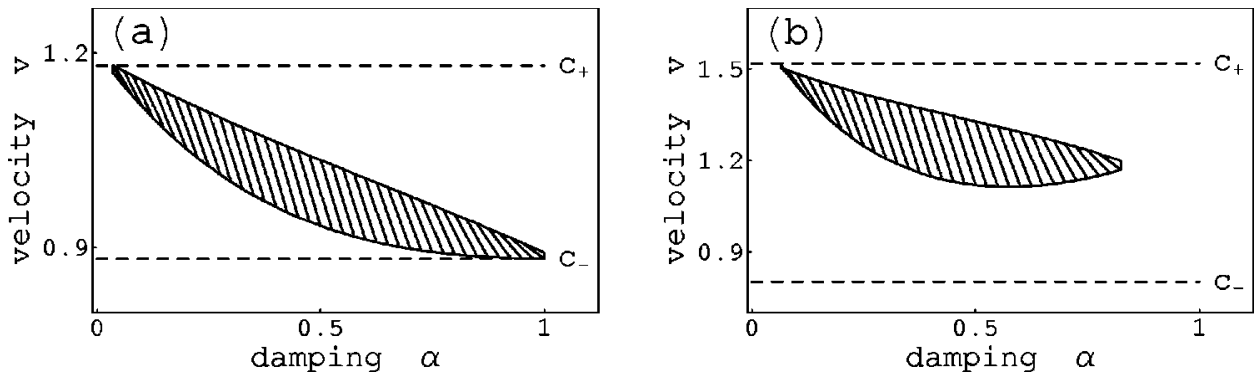

FIG. 4. Dashed regions indicate ranges of the fluxon velocity $v$ and damping coefficient $\alpha$, where the bunched state exists. Coupling constant $S=-0.2$ (a) and $S=-0.4(\mathrm{~b})$.
A symmetric central finite-difference method of second order for both space and time has been implemented for the numerical simulations. The total length of the junctions is $L=40$ and the spatial mesh size is $\Delta x=0.05$. We have chosen periodic boundary conditions $\varphi_{i}(L / 2)=\varphi_{i}(-L / 2)+2 \pi$ and $\varphi_{i, x}(L / 2)=\varphi_{i, x}(-L / 2), i=1,2,3$, corresponding to an annular geometry to avoid ambiguities due to reflection from edges.

The fluxon-fluxon-fluxon initial condition may lead to three possible dynamical states (see Fig. 3). Whenever the velocity induced in the fluxons by $\gamma$ is lower than $c_{-}$, then the fluxons split. $\varphi_{2}$ will travel with velocity $v_{2}$, faster than the other two identical fluxons $\varphi_{1}=\varphi_{3}$ which travel with velocity $v_{1}$. Experiments with two ${ }^{17}$ and three ${ }^{18}$ junctions confirm the fluxon splitting.

When sufficiently high bias current drives the fluxons with speed exceeding the Swihart velocity the fluxons may bunch, as illustrated in Fig. 3(b). This phenomenon was first observed numerically for multiple fluxons in one junction in Ref. 19. The reason for bunching is that the dispersion equation for the plasma waves has complex roots which leads to the existence of oscillating tails. These oscillations have opposite polarity in adjacent junctions and their contribution to $U_{\text {int }}$ is negative, giving rise to an attraction between the fluxons. In contrast to this the interaction between the central part of the fluxons is repulsive. The balance between these two contributions will determine the relative position of the fluxons in the stable state at a local minimum of the potential energy $W$. Only for a certain range of speeds, above the lowest characteristic velocity, $c_{-}$, will $W$ have this local minimum and bunching occurs. The phase diagram presented in Fig. 4 is obtained by changing the bias $\gamma$ for fixed values of $\alpha$ and $S$. The contour separating the dashed region corre- sponds to dynamical states where bunching ceases to exist. It is worth noting that transversing the bottom contour, the velocities of all three fluxons become less than $c_{-}$. On the other hand, bunching of unlocked fluxons by increasing the bias is not possible. This phenomenon does not occur in a single junction, unless surface current losses $(\beta \neq 0)$ are included in Eq. (1). ${ }^{20-25}$

Returning to Fig. 3 we see that for high bias $[\gamma=0.69$ in Fig. 3(c)] a new pair of fluxons and antifluxons is created in the same manner as in Ref. 26.

The two-junction case has been extensively investigated and bunching of fluxons has been attributed to emission of Cherenkov radiation. ${ }^{7,27-29}$ The stability of the bunched state for velocities $v$, where $c_{-}<v<c_{+}$, was investigated by means of perturbation analysis of the antiphase linear mode by Grønbech-Jensen et al. ${ }^{9,11,30}$ Numerical simulations showing the attraction between fluxons due to radiation emission in several junctions have been made. ${ }^{6}$ The results concerning bunched dynamics of fluxons are summarized in Ref. 31.

In fact one fluxon in one of the junctions does have an oscillatory tail and induces radiation in the other junctions and vice versa. ${ }^{4}$ In the case of two identical junctions a combination of two fluxons results in a nonoscillatory tail because of exact cancellation of antiphase oscillations. As shown in Fig. 1(b) of Ref. 4 the fluxon tail and the induced radiation are mirror symmetric. In the case of slightly different junctions, the cancellation is incomplete and this results in oscillatory tails in both junctions.

Figure 5 plots the bias current versus the numerically found fluxon velocities (i.e., an $I-V$ curve with voltage replaced by velocity). When the fluxons are driven slower than $c_{-}$, they split and travel with different velocities $\varphi_{1}=\varphi_{3}$ with $v_{1}$ and $\varphi_{2}$ with $v_{2}$, where $v_{1}<v_{2}$. Bunching-state

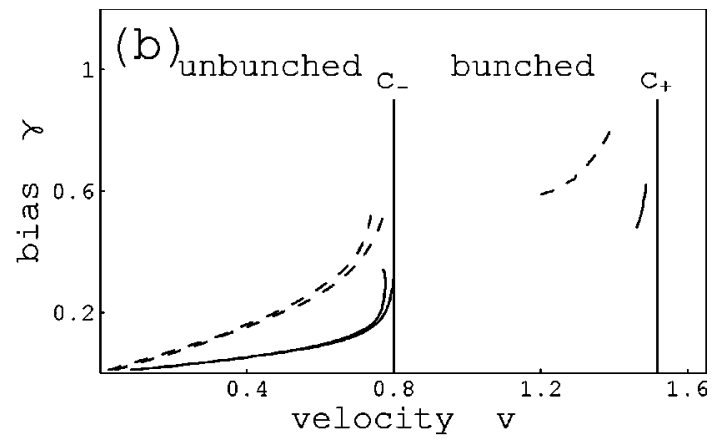

FIG. 5. Bias current $\gamma$ vs fluxon velocity $v$ for coupling (a) $S=-0.2$ and (b) $S=-0.4$. Solid (dashed) curves represent velocity vs bias for $\alpha=0.1(\alpha=0.3)$. Below $c_{-}$, fluxons split and two different velocity branches are observed for $\varphi_{1}$ and $\varphi_{2}$. Fluxon bunching occurs in a velocity interval between $c_{-}$and $c_{+}$. 
branches are observed in narrow ranges of velocities between $c_{-}$and $c_{+}$. No phase change is shown in the vicinity of the velocity $c_{d}$ corresponding to the decoupled mode $\mathrm{f}-0$-a. The reason is that this last mode cannot be excited by a unidirectional external bias current $\gamma$, where the junctions of the top and bottom of the stack will develop fluxons of equal polarity.

We have studied numerically, using periodic boundary conditions, the behavior of the fluxon-fluxon-fluxon state subject to different external bias current and dissipation. Whenever the balance between bias and damping provides a fluxon velocity $v=v_{1}=v_{2}$ higher than the lowest Swihart velocity $c_{-}$, oscillating tails appear and their interaction may overcome the repulsion between the central parts of the fluxons. The balance between the attraction and repulsion results in a bunched state of fluxons in a narrow region of the parameter space $(\alpha, v)$.

C.G. and Yu.B.G. acknowledge the hospitality of the Technical University of Denmark, where the investigations have been performed. Financial support was provided by the Marie Curie Training Site program of the EU (No. HPMTCT-2001-00402) and a project from the Basque Country University (UPV/EHU 100.310-E-13891/2001).
${ }^{1}$ D.W. McLaughlin and A.C. Scott, Phys. Rev. A 18, 1652 (1978).

${ }^{2}$ S. Sakai, P. Bodin, and N.F. Pedersen, J. Appl. Phys. 73, 2411 (1993).

${ }^{3}$ M.B. Mineev, G.S. Mkrtchyan, and V.V. Schmidt, J. Low Temp. Phys. 45, 497 (1981).

${ }^{4}$ E. Goldobin, B.A. Malomed, and A.V. Ustinov, Phys. Rev. B 62, 1414 (2000).

${ }^{5}$ E. Goldobin, A. Wallraff, and A.V. Ustinov, J. Low Temp. Phys. 119, 589 (2000).

${ }^{6}$ A.V. Ustinov and S. Sakai, Appl. Phys. Lett. 73, 686 (1998).

${ }^{7}$ Y.S. Kivshar and B.A. Malomed, Phys. Rev. B 37, 9325 (1988).

${ }^{8}$ G. Carapella, G. Costabile, A. Petraglia, N.F. Pedersen, and J. Mygind, Appl. Phys. Lett. 69, 1300 (1996).

${ }^{9}$ N. Grønbech-Jensen, D. Cai, and M.R. Samuelsen, Phys. Rev. B 48, 16160 (1993).

${ }^{10}$ G. Carapella, G. Costabile, R. Latempa, G. Filatrella, and J. Mygind, Phys. Rev. B 62, 9095 (2000).

${ }^{11}$ N. Grønbech-Jensen, D. Cai, A.R. Bishop, A.W.C. Lau, and P.S. Lomdahl, Phys. Rev. B 50, 6352 (1994).

${ }^{12}$ J.C. Swihart, J. Appl. Phys. 32, 461 (1961).

${ }^{13}$ S. Sakai, A.V. Ustinov, H. Kohlstedt, A. Petraglia, and N.F. Pedersen, Phys. Rev. B 50, 12905 (1994).

${ }^{14}$ N.F. Pedersen, Supercond. Sci. Technol. 15, 405 (2002).

${ }^{15}$ A. Petraglia, A.V. Ustinov, N.F. Pedersen, and S. Sakai, J. Appl. Phys. 77, 1171 (1995).

${ }^{16}$ N.F. Pedersen and S. Sakai, Phys. Rev. B 58, 2820 (1998).
${ }^{17}$ A.V. Ustinov and H. Kohlstedt, Phys. Rev. B 54, 6111 (1996).

${ }^{18}$ A.V. Ustinov, H. Kohlstedt, M. Cirillo, N.F. Pedersen, G. Hallmanns, and C. Heiden, Phys. Rev. B 48, 10614 (1993).

${ }^{19}$ A. Davidson, N.F. Pedersen, and S. Pagano, Appl. Phys. Lett. 48, 1306 (1986).

${ }^{20}$ P.L. Christiansen, P.S. Lomdahl, A.C. Scott, O.H. Soerensen, and J.C. Eilbeck, Appl. Phys. Lett. 39, 108 (1981).

${ }^{21}$ P.S. Lomdahl, O.H. Soerensen, P.L. Christiansen, A.C. Scott, and J.C. Eilbeck, Phys. Rev. B 24, 7460 (1981).

${ }^{22}$ S. Pagano, M.P. Soerensen, P.L. Christiansen, and R.D. Parmentier, Phys. Rev. B 38, 4677 (1988).

${ }^{23}$ B.A. Malomed, Phys. Rev. B 47, 1111 (1993).

${ }^{24}$ M.P. Soerensen, B.A. Malomed, A.V. Ustinov, and N.F. Pedersen, Physica D 68, 38 (1993).

${ }^{25}$ I.V. Vernik, N. Lazarides, M.P. Soerensen, A.V. Ustinov, N.F. Pedersen, and V.A. Oboznov, J. Appl. Phys. 79, 7854 (1996).

${ }^{26}$ M. Peyrard and D. Kruskal, Physica D 14, 88 (1984).

${ }^{27}$ N. Grønbech-Jensen, M.R. Samuelsen, P.S. Lomdahl, and J.A. Blackburn, Phys. Rev. B 42, 3976 (1990).

${ }^{28}$ E. Goldobin, A. Wallraff, N. Thyssen, and A.V. Ustinov, Phys. Rev. B 57, 130 (1998).

${ }^{29}$ A. Wallraff, E. Goldobin, and A.V. Ustinov, J. Appl. Phys. 80, 6523 (1996).

${ }^{30}$ N. Grønbech-Jensen, P.S. Lomdahl, and M.R. Samuelsen, Phys. Rev. B 48, 6353 (1993).

${ }^{31}$ A.V. Ustinov, Physica D 123, 315 (1998). 